\title{
A formulation of Clausena anisata (Willd.) Hook. f. Ex Benth and Cassia sieberiana DC. alleviates the symptoms associated with osteoarthritis: a single-blind, randomised controlled trial of a traditional Ghanaian remedy
}

Kwesi Prah Thomford ${ }^{1,2^{*}}$ (D) J Joseph Yorke ${ }^{3}$, Ama Kyeraa Thomford ${ }^{4}$ (D) and Isaac Kingsley Amponsah ${ }^{5}$ (D)

\begin{abstract}
Background: Clinical validation of herbal medicinal products is important for their widespread acceptance and application. In this single-blind, randomised controlled trial, a traditional Ghanaian herbal medicine formulation with Clausena anisata (Willd.) Hook. f. ex Benth and Cassia sieberiana DC. as ingredients was evaluated for its safety and effectiveness in the management of patients with osteoarthritis. The formulation for the purposes of this study was labelled with the code GC-500.

Methods: A total of 57 participants were randomly assigned to receive either this herbal remedy, GC-500, or the control treatment of diclofenac. Subjects were then followed up for a period of 8 weeks using a modified Graded Chronic Pain Scale (GCPS) for their efficacy assessments. The control treatment comprised 13 subjects and the GC500 group 44 subjects.

Results: Upon completion of the study, 28 (63.63\%) subjects in the GC-500 group attained the primary outcome, (GCPS of Grade 0) compared to 5 (38.46\%) subjects in the control group. Improvement in disease indicators such as characteristic pain intensity, disability score and disability days was comparable between the GC-500 and diclofenac. Intensity of pain reduced after 8 weeks of treatment; disability score and disability days also declined indicating an improvement in the quality of life of subjects. GC-500 was also shown to be safe for human use.
\end{abstract}

Conclusion: The herbal medicine formulation GC-500, provides a credible treatment option for managing the pain associated with osteoarthritis.

\footnotetext{
* Correspondence: kwesi.thomford@ucc.edu.gh;

kpthomford.chs@knust.edu.gh

'Department of Pharmacognosy and Herbal Medicine, School of Pharmacy and Pharmaceutical Sciences, University of Cape Coast, Cape Coast, Ghana

${ }^{2}$ Department of Herbal Medicine, Faculty of Pharmacy and Pharmaceutical Sciences, Kwame Nkrumah University of Science and Technology, Kumasi,

Ghana

Full list of author information is available at the end of the article
}

\section{Springer Open}

(c) The Author(s). 2021 Open Access This article is licensed under a Creative Commons Attribution 4.0 International License, which permits use, sharing, adaptation, distribution and reproduction in any medium or format, as long as you give appropriate credit to the original author(s) and the source, provide a link to the Creative Commons licence, and indicate if changes were made. The images or other third party material in this article are included in the article's Creative Commons licence, unless indicated otherwise in a credit line to the material. If material is not included in the article's Creative Commons licence and your intended use is not permitted by statutory regulation or exceeds the permitted use, you will need to obtain permission directly from the copyright holder. To view a copy of this licence, visit http://creativecommons.org/licenses/by/4.0/. 
Trial registration: This study was retrospectively registered with the Pan-African Trial registry with a Trial ID:

PACTR201909643671755 on 25th September, 2019 at https://pactr.samrc.ac.za/Search.aspx

Keywords: Clausena anisata (Willd.) Hook. f. Ex Benth, Cassia sieberiana DC., Osteoarthritis, Herbal medicines, Randomised controlled trial

\section{Introduction}

Osteoarthritis involves damage to articular cartilage and other structures in and around joints, with a variable degree of inflammation and repair $[1,2]$. The prevalence of the condition is predicted to rise as the life expectancy of the general population in many countries also increases [3]. The disease should be of public health interest because of its burden on patients with regards to disability, economic hours lost and the overall impact on society.

Non-steroidal anti-inflammatory drugs (NSAIDs) are extensively used for the treatment of pain and inflammation in patients with osteoarthritis [4-6]. However, their use is associated numerous side effects. One of the most common side effects is the damage caused to the gastrointestinal mucosae partly because of a block in the production of protective prostaglandins synthesised via cyclo-oxygenase (COX) pathway [7, 8]. When other treatment options are available to patients, factors such as cost of treatment, risk of addiction and long-term damage to organs like the kidneys are hurdles physicians need to handle and overcome.

This challenge with conventional treatments for osteoarthritis is responsible for the renewed interest in complementary and alternative medicines. In Ghana and other African countries, such remedies are a key component in the management of pain and its related disorders [9]. The advantage here is their relatively low toxicity and high tolerance. Despite these positives, the integration of alternative anti-arthritic medicines, and herbal remedies in general, into the conventional system of health care has remained limited due to the lack of scientific evidence to support their safety and effectiveness.

We report on the clinical evaluation of a traditional Ghanaian herbal formulation coded GC-500. The product is indicated for the management of pain, dysmenorrhoea and abdominal upsets. Its ingredients comprise the roots of Clausena anisata (Willd.) Hook. f. ex Benth and Cassia sieberiana DC. prepared according to a traditional formula.

Clausena anisata (Willd.) Hook. f. ex Benth (syn. Clausena abyssinica (Engl.) Engl., Clausena inequalis (DC.) Benth.) is an evergreen shrub in the Rutaceae family that grows abundantly throughout Africa. In the Akan language of Ghana, it is known as samandua. Traditionally, various parts of this plant are reported to be effective for the treatment of rheumatism, malaria, heart disorders, hypertension and other inflammatory conditions [10, 11]. Cassia sieberiana DC. (syn. Cassia kotschyana Oliv.; Senna sieberiana DC.), the other plant in the product, also belongs to the family LeguminosaeCaesalpinioideae. It is found in the savannah forests, thickets and costal shrubs of Ghana. Traditional practitioners have employed various parts of the plant for the treatment of abdominal pains, malaria, feverishness and toothache $[12,13]$. The indications listed for the 2 plants have not been clinically verified hence its use is still based on folkloric knowledge. The primary objective of this trial was thus to establish the clinical efficacy of $G C$ 500. The clinical safety of the product and its effect on the quality of life (QOL) indicators of users were also considered as secondary objectives.

\section{Materials and methods}

\section{Chemicals and reagents}

Solvents used: methanol $(\mathrm{MEOH})$, acetonitrile $(\mathrm{ACN})$, acetic acid $\left(\mathrm{CH}_{3} \mathrm{COOH}\right)$ and tetrahydrofuran (THF) were of HPLC grade (Sigma Aldrich, St. Louis, USA). Deionised water was prepared by a Milli-Q Water purification system (Millipore, MA, USA). The standard compound, quercetin, were also purchased from SigmaAldrich, USA. Polytetrafluoroethylene (PTFE) syringe membrane filters employed during the HPLC were also purchased from Thermo Fischer Scientific, Massachusetts, USA. All other reagents used in testing were of laboratory grade.

\section{Methods \\ Plant material extraction, product formulation and quality assessment \\ Plant materials and extraction}

The classical manufacturing method was refined by a process that involved the collection of the plant materials, verification by Dr. George Henry Sam, a Botanist with the Department of Herbal Medicine, preparation of an herbarium sample and assignment of the specimen numbers KNUST/HM1/09/L 027 and KNUST/HM1/12/ L031 for C. sieberiana and C. anisata respectively. Plant materials were then washed, dried under shade for 7 days and milled using a hammer mill. The powdered plant materials were extracted with industrial grade ethanol $(98.0 \% \mathrm{v} / \mathrm{v})$ for $72 \mathrm{~h}$. The resultant extract is then dried into a thin layer of concentrate in a hot oven at temperatures between 50 and $60^{\circ} \mathrm{C}$ for $48 \mathrm{~h}$. Materials 
were stored in a desiccator prior to formulation. Yields obtained were $C$. anisata: $5.33 \%(\mathrm{w} / \mathrm{w})$ and $C$. sieberiana: $7.04 \%\left({ }^{\mathrm{w}} / \mathrm{w}\right)$.

\section{Formulation of GC-500}

Granules for encapsulation were formulated by uniformly mixing $20\left({ }^{\mathrm{w}} / \mathrm{w} \%\right)$ of C. sieberiana and C. anisata together with $20\left({ }^{\mathrm{w}} / \mathrm{w} \%\right)$ of locally sourced maize starch, drying in a hot oven, sieving through an $850 \mu \mathrm{m}$ mesh and then packing into $500 \mathrm{mg}$ hard gelatin shells using a manual encapsulating machine. The manufacturing process was performed according to a standard protocol developed by the Department of Herbal Medicine and met current Good Manufacturing Practices (cGMP).

\section{Standardisation of the formulation GC-500}

GC-500 was standardised using a High-Performance Liquid Chromatography (HPLC) and a basic phytochemical screening. The HPLC involved the development and validation of a suitable method for the assay quercetin (Sigma-Aldrich, St. Louis, USA). The quercetin content in the product was assayed as an analytical marker for the product. This marker was selected based on availability and its diverse bioactivity that includes its antioxidant, anti-inflammatory and significance in the prevention and management of chronic painful arthritic conditions. Validation was conducted per the guidelines of the International Conference for Harmonisation (ICH) [14].

Chromatographic conditions for the analysis comprised a Dionex Ultimate 3000 RS Pump HPLC, degasser, autosampler Dionex Ultimate XRS and a Dionex Ultimate RS diode array multiple wavelength detector. The column used was a BDS Hypersil $C_{18}$ reverse phase column (120A pore size, $\varnothing 4.6 \mathrm{~mm} \times 150 \mathrm{~mm}$ ). The mobile phase employed was Acetonitrile (ACN), methanol $(\mathrm{MeOH})$ and $1 \%$ acetic acid $\left(\mathrm{CH}_{3} \mathrm{COOH}\right)$ (15: 40: 45) with a flow rate of $1.0 \mathrm{ml} / \mathrm{min}$ and injection volume of $20 \mu \mathrm{l}$. Column temperature was also kept at an ambient temperature of $26^{\circ} \mathrm{C}$. The wavelength for detection was set at $365 \mathrm{~nm}$. Standard stock solution $(1 \mathrm{mg} / \mathrm{ml})$ of the reference compound, quercetin, was prepared in methanol. Calibration and validation of the method was achieved by triplicate injection of ten (10) different concentrations of the reference in the range of $1 \mathrm{mg} / \mathrm{ml}-$ $3.9 \times 10-5 \mathrm{mg} / \mathrm{ml}$ post a twofold serial dilution of the stock solution. In preparing the samples for HPLC analysis, the plant extracts and herbal product GC-500 obtained as reported in section 2.2.1 were reconstituted in methanol, in triplicate, to attain a concentration of 1 $\mathrm{mg} / \mathrm{ml}$. Samples were then filtered through a through a $0.45 \mu \mathrm{m}$ PTFE membrane syringe filter (Thermo Fischer, Massachusetts, USA) prior to injection. Basic phytochemical evaluation was undertaken according to the methods described by Gul and his colleagues [15].

\section{Clinical study \\ Ethical considerations}

The protocol used for the study, consent form and the patient information sheet were reviewed and approved by the human experimentation committee of the Legacy Specialist Hospital, Kumasi - Ghana (LSH/ME/002/01) prior to the initiation of the study. The study was conducted in accordance with the Declaration of Helsinki and Good Clinical Practice [16]. Written informed consent from every study subject was obtained prior to the trial-related activities; the consent forms were retained by the investigators. The general guidance provided for human studies were also given to participants. These included, but was not limited to, the freedom to withdraw at any stage of the study, protection of their private data and their right to standard care if they opt out of the study.

\section{Study site}

The study was conducted at the out-patient unit of the Legacy Specialist Hospital, Kumasi, Ghana. This hospital comprises an out and in-patient unit manned by two (2) specialists and two (2) general practitioners. The hospital through the facilitation of the Department of Herbal Medicine, KNUST has been partnering with some traditional medicine practitioners in Ghana to improve their practice. This partnership is part of the wider framework of integrating herbal medicines into mainstream medical practices in the country.

\section{Study design}

The study was designed as a prospective, single blind, randomised controlled trial comparing the formulation GC-500 to the standard analgesic diclofenac sodium (control). Protocol and sequence of the trial is illustrated as Fig. 1.

\section{Randomisation, blinding and subject allocation}

A blocked randomisation was used in assigning subjects to the treatment and the control groups. Assignment was done in a ratio of 2:1 i.e. GC-500 and the control respectively. To achieve randomisation, participants were asked to pick, without replacing, from a box that contained 15 folded papers with 10 labelled for the herbal treatment and 5 for the control. Thus, at the end of each round of recruitment and randomisation, the herbal treatment would have 10 participants for the herbal medicinal product and 5 participants for the control group (Fig. 1). This allocation ratio was chosen because of the need to gather evidence on the safety and risk of adverse events for $G C-500$. 


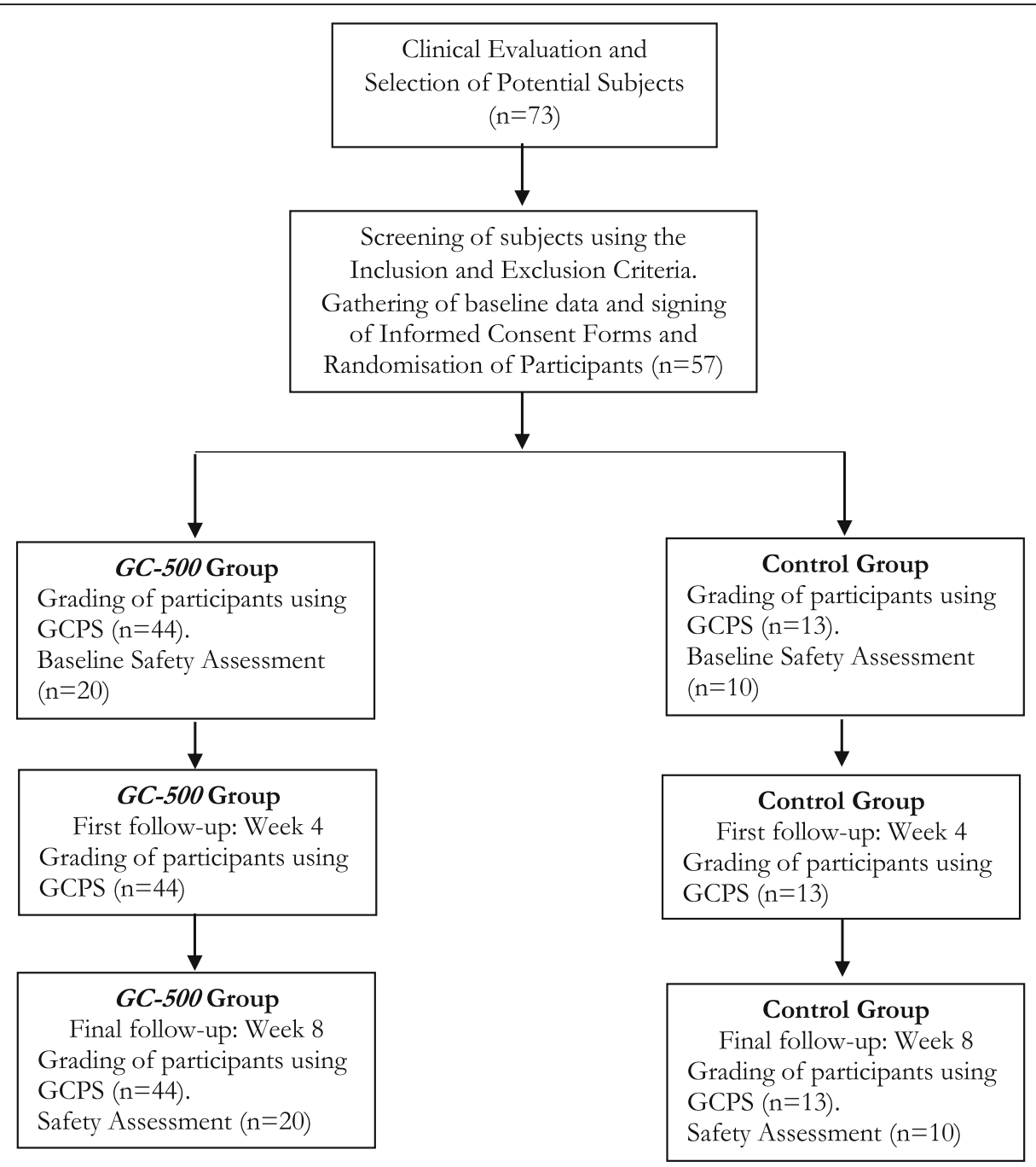

Fig. 1 Schematic representation of the sequence of activities participants were involved, randomisation groups for the study

Clinicians responsible for subject evaluations were blind to the treatment received by the participants in the trial. This single-blind method was used because of the organoleptic nature of the herbal formulation, GC-500. The characteristic colour, taste and odour of the ingredients made it difficult to design a comparable placebo. Blinding of clinicians was ensured as they were not involved at any point in the assignment or dispensing of treatment.

\section{Eligibility criteria}

Participants were classified as eligible if they were male or female between the ages of 45 to 75 years and clinically diagnosed with a chronic pain from an osteoarthritis. These participants should not have been on any analgesic or anti-inflammatory agents for a minimum of 4 weeks. The criteria for the clinical diagnosis of osteoarthritis comprised: a history of primary idiopathic osteoarthritis of any joint characterised by pain of mild to moderate intensity and requiring the intake of analgesics, recurrent pain in any of the major joints for more than 6 months with patient-reported or physicianidentified difficulty in undertaking at least one of the following activities due to the pain: limitation in the range of motion, difficulty in performing routine activities that require the use of the joint e.g. walking over a distance of 200-500 m, going up and down a flight of stairs, early morning stiffness and stiffness observed in the joint after maintaining a posture for a short period. Radiological confirmation was undertaken for subjects being diagnosed for the first time.

Exclusion criteria comprised individuals whose pain were of metastatic origin and those receiving an opioid or steroid based treatment. Participants were also to be free of any clinically significant diseases such as an endocrine, gastrointestinal, autoimmune disorders and a pre- 
existing cardiovascular risk. Subjects were required to be willing to adhere to the protocol and provide an informed consent. Individuals with hepatic and renal impairment as well as the acutely ill were all excluded from the study.

Participants were withdrawn if there was an increase in the GCPS compared to the baseline, developed any adverse reaction or decided at any point they did not want to continue with the study. Such participants were transferred to a physician for standard care at no cost to them.

\section{Study outcomes}

The primary outcome of this study was a reduction in the baseline disease score (Graded Chronic Pain Scale) to Grade 0 by the end of the 8th week for all participants. This meant that after a treatment cycle of 4 weeks, subjects should report with no pain over the prior month, a maximum of 3 days of disability and disability score of $<30$ points. Secondary outcomes of interest were the ability of the product to improve the physical and socioeconomic impact of the disease represented by a reduction in either the Characteristic Pain Intensity and/or a decline in the Disability Point.

\section{Assessment tool}

The tool for monitoring the effectiveness of the treatments was the Graded Chronic Pain Scale (GCPS) with modifications for this study [17]. Modifications were made to the length of time for the recall of disease impact and pain: from 6 months to 3 months at baseline assessment and a 1 month recall during follow ups. The Visual Analogue Scale (VAS) was also used as an aid during scoring by participants. The GCPS comprises 7 questions with respondents choosing from a 10-point scale for each question. Questions 1-3 aid in the characterisation of the intensity of the pain the subject is experiencing (Characteristic Pain Intensity). Questions 4-6 cover the impact of pain on the physical, socioeconomic and quality of life of subjects (Disability
Score). Question 7 is a query about the number of days lost over the past month due to the pain (Disability Days). The Disability Score and Disability Days are then transformed using a 4-point system and the two (2) scores summed up to attain the Disability Point. The sum of the Characteristic Pain Intensity and the Disability Days is also graded between 0 - IV (Fig. 2).

\section{Safety assessment}

Safety evaluation was performed using the test values of creatinine, urea, sodium $\left(\mathrm{Na}^{+}\right)$, potassium $\left(\mathrm{K}^{+}\right)$and chloride $\left(\mathrm{Cl}^{-}\right)$for kidney function; alanine aminotransferase (ALT), aspartate transaminase (AST), alkaline phosphatase (ALP), $\gamma$-glutamyl transaminase (GGT), lactate dehydrogenase (LDH), total bilirubin (TBIL), total protein (TPROT) and albumin (ALB) for liver function. Haematological assessment was also determined using the white blood cell count (WBC), red blood cell (RBC), haematocrit (HCT), haemoglobin (HGB) and platelet counts (PLT). Additionally, an active surveillance of harms was undertaken to identified related adverse effects using the standardised World Health Organisation questionnaire.

\section{Herbal intervention}

The herbal medicine formulation, $G C-500$ is a prepared from the dried roots of $C$. sieberiana and $C$. anisata. This formulation has been in use for decades by some traditional practitioners in Ghana. Classically, the product is formulated either as a decoction or an ethanolic extract to be administered twice daily. A relatively improved formulation of this remedy also available on the Ghanaian market involves the filling of $500 \mathrm{mg}$ gelatin capsules with dry pulverised plant materials which is administered by patients twice a day.

\section{Control treatment}

The control drug was the non-steroidal antiinflammatory (NSAIDs) agent Diclofenac sodium which is currently indicated for pain associated with

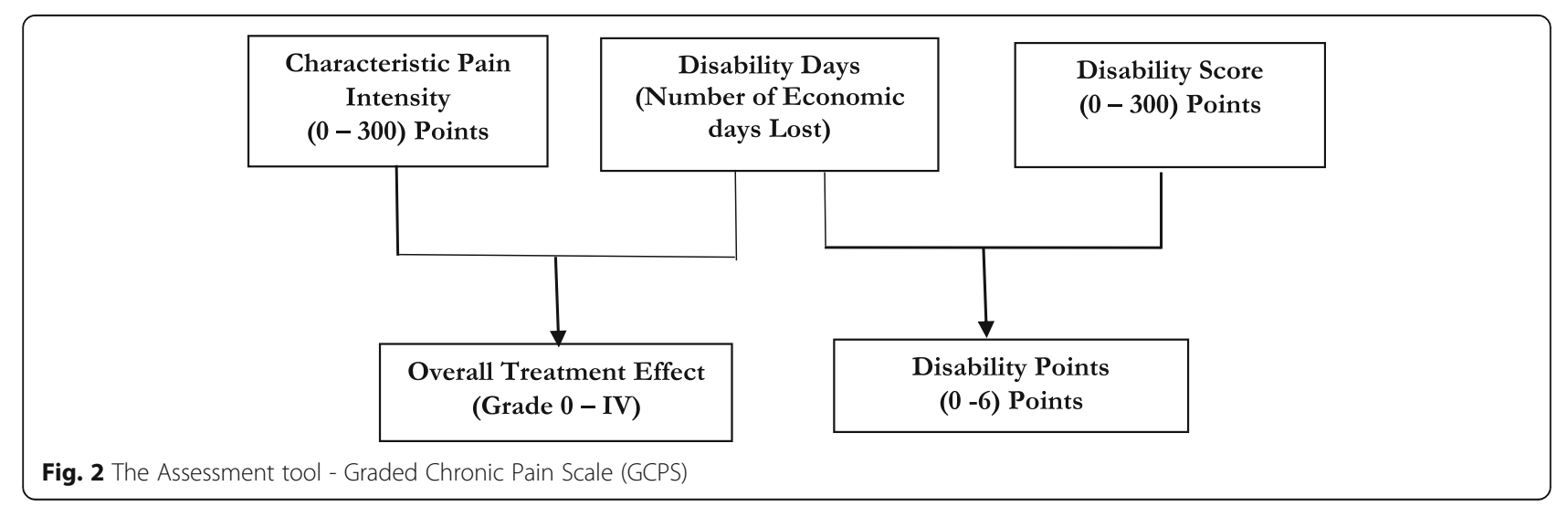


inflammatory diseases. Subjects randomised to the control treatment received $50 \mathrm{mg}$ of Diclofenac twice a day while those recruited into the treatment group received $500 \mathrm{mg}$ of the herbal formulation twice daily. All treatments were administered for a period of 8 weeks and participants were advised to use only their assigned treatments for the management of the condition.

\section{Statistical analysis}

We hypothesised that in this study, the herbal formulation would have better or comparable activity to that of diclofenac. A sample size of 60 participants was calculated to be ideal for testing this outcome. This sample size calculation was performed taking cognisance of the fact that the study should have an $80 \%$ probability of detecting treatment differences between the two groups, if the true difference between treatments was at least 0.5 times the standard deviation. Calculation of the sample size was undertaken using an online tool provided by David Schoenfeld [18] . Safety analysis was also performed using an independent $t$-test comparing the herbal treatment group to the control. An $\alpha$-level of 5\% was set for the detection of all statistically significant differences.

All analyses were done using the intention to treat (ITT) population. Column statistics followed by a oneway analysis of variance (ANOVA) with a Bonferroni posthoc-test was used to represent and compare the differences between the herbal treatment and the control groups. All data was analysed using GraphPad Prism version 9.0 software and is presented as mean $( \pm \mathrm{SD})$.

\section{Results}

\section{Quality assessment}

\section{Standardisation of GC-500}

The product, $G C-500$, was quantified as having a quercetin content of $0.31( \pm 0.08) \% \mathrm{w} / \mathrm{w}$. In the case of the starting materials, C. sieberiana had a quercetin content of $0.11( \pm 0.03) \%{ }^{\mathrm{w}} / \mathrm{w}$ and C. anisata $0.502( \pm 0.03) \% \mathrm{w} /$ w. Chromatographic fingerprint from the HPLC analysis for the product is indicated as Fig. 3. The basic phytochemical screening of the product conducted also indicated the presence of phenolics, flavonoids, tannins, anthraquinone glycosides, and phytosterols.

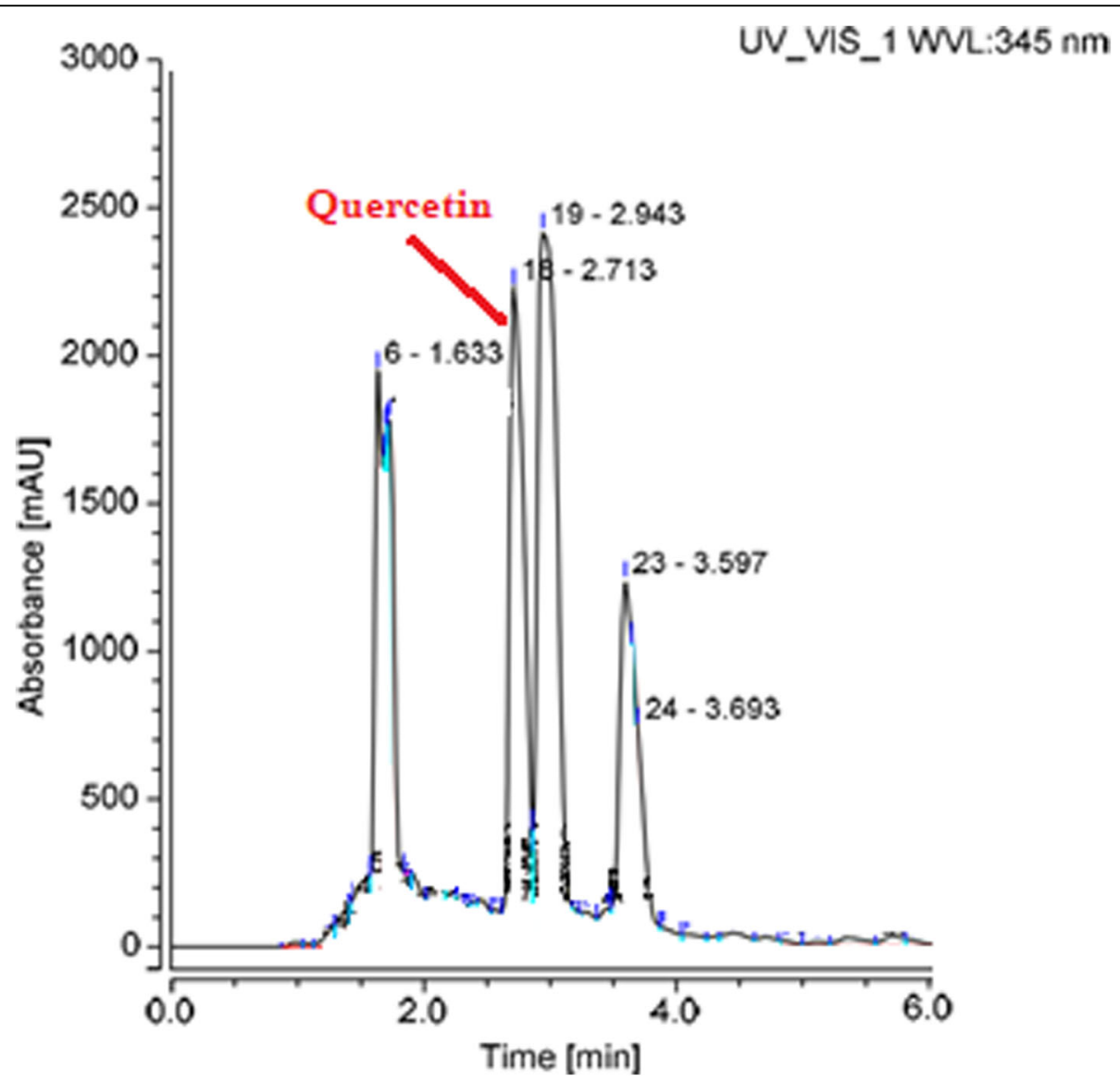

Fig. 3 Qualitative HPLC fingerprint for GC-500. Quercetin appears as peak No 18 at a time of 2.713 min. Quercetin content in the product was $0.31( \pm 0.08) \% \mathrm{w} / \mathrm{w}$ 


\section{Clinical study}

\section{Patient demographics}

A total of 44 participants in the treatment group and 13 in the control group qualified to be included in the analysis after randomisation. The mean age of participants was $65.8( \pm 3.12)$ years and $64.3( \pm 4.12)$ years for the treatment and control groups respectively. The most recorded form of osteoarthritis was of the knee joint and diagnosed in $50(87.77 \%)$ of the subjects. Osteoarthritis of the shoulder joint was also identified in 4 (7.02\%) of the subjects while $3(5.26 \%)$ of subjects reported of ankle, wrist and elbow disorders. Summary of the demographics of subjects are reported in Table 1.

\section{Effectiveness of the herbal medicine, GC-500}

Out of the 44 patients in the GC-500 group, 11 (25\%) of them achieved the primary outcome of Grade 0 by week 4 and $28(63.63 \%)$ at the end of the study (Table 2). A relatively lower outcome was observed in the control group with $5(38.46 \%)$ participants achieving the primary outcome by week 4 and week 8 . Overall, there was some clinical improvement attributable to the effect of the test products across both groups throughout the study. This pattern of change in disease outcome was seen in the number of participants who saw a reduction in their overall disease grading.

Analysis of the data also indicated specific reduction in the physical and socio-economic burden (Disability Scores) among subjects. By weeks 4 and 8, the mean baseline disability score of 101.4 ( \pm 9.049) was reduced significantly to $46.36( \pm 5.898)$ and $25.23( \pm 3.339)$ respectively for the treatment group (Fig. 4). There was also a significant reduction in the disability score of the subjects in the control group: 25.83 ( \pm 5.288). At the end of the 8-week study, the GC-500 group also achieved a similar decline in their disability points compared to the control group (Fig. 5).

The specific effect of the treatment on characteristic pain intensity of subjects during the study was also recorded as a secondary measure of effectiveness. Subjects in the treatment group reported a significant reduction in the intensity of pain with a mean reduction from $205.9( \pm 4.065)$ at baseline to $90( \pm 3.75)$ and $47.05( \pm$

Table 1 Demographics of subjects in this study

\begin{tabular}{lll}
\hline & GC-500 & Control \\
\hline Age & $65.8( \pm 3.12)$ & $64.3( \pm 4.12)$ \\
Sex: & & \\
$\quad$ Males n (\%) & $12( \pm 27.27 \%)$ & $5( \pm 38.46 \%)$ \\
Females n (\%) & $32( \pm 72.72 \%)$ & $8( \pm 61.54 \%)$ \\
BMl & $26.32( \pm 4.02)$ & $24( \pm 2.31)$ \\
\hline
\end{tabular}

Data is presented as $n(\%)$ \& Mean $( \pm$ SD); $N=57$ : GC-500 ( $n=44)$ \& Control $(n=13) . p>0.05$ after statistical testing for age and BMI.
Table 2 Summary of the Overall Effect of the Treatments using the 5-point Grading System (Primary Outcome)

\begin{tabular}{|c|c|c|c|c|c|c|}
\hline & \multicolumn{2}{|l|}{ Baseline } & \multicolumn{2}{|l|}{ Week 4} & \multicolumn{2}{|l|}{ Week 8} \\
\hline & GCP-500 & Control & GCP-500 & Control & GCP-500 & Control \\
\hline Grade 0 & - & - & $11(25.0)$ & $\begin{array}{l}5 \\
(38.46)\end{array}$ & $\begin{array}{l}28 \\
(63.63)\end{array}$ & $\begin{array}{l}5 \\
(38.46)\end{array}$ \\
\hline Grade I & $3(6.81)$ & $\begin{array}{l}3 \\
(23.07)\end{array}$ & $\begin{array}{l}19 \\
(43.18)\end{array}$ & $\begin{array}{l}6 \\
(46.15)\end{array}$ & $\begin{array}{l}13 \\
(29.54)\end{array}$ & $\begin{array}{l}6 \\
(46.15)\end{array}$ \\
\hline Grade II & $6(13.63)$ & $\begin{array}{l}5 \\
(38.46)\end{array}$ & $6(13.63)$ & $\begin{array}{l}2 \\
(15.38)\end{array}$ & $1(2.20)$ & $\begin{array}{l}2 \\
(15.38)\end{array}$ \\
\hline Grade III & $\begin{array}{l}32 \\
(72.72)\end{array}$ & $\begin{array}{l}5 \\
(38.46)\end{array}$ & $6(13.63)$ & - & $1(2.20)$ & - \\
\hline $\begin{array}{l}\text { Grade } \\
\text { IV }\end{array}$ & $3(6.81)$ & - & $2(4.50)$ & - & $1(2.20)$ & - \\
\hline
\end{tabular}

Data is presented as $\mathrm{n}(\%) ; N=57: \mathrm{GC}-500(n=44)$ \& Control $(n=13)$.

3.244) at week 4 and 8 respectively. Comparable change was recorded for the control group with mean baseline Characteristic Pain Intensity of 196.7 (6.779) which declined to $71.67(9.115)$ at the end of the study (Fig. 6).

\section{Safety assessment of the investigational product}

There was no reported adverse effect by any of the participants neither was any detected by the investigator throughout the 8 weeks of study. An active surveillance of harm was used. At the end of the study, the participants showed no significant changes in baseline indices for liver such as AST, ALP, ALB and GGT (Table 3). Renal indices such as electrolytes, urea and creatinine, together with haematological variables implicated in organ damage from the use of medicinal products were also normal (Table 4). No drug related toxicity was observed in all participants from the study.

\section{Discussion}

GC-500 is a traditional Ghanaian herbal medicine used by some traditional medicine practitioner in Ghana. The product has the roots of $C$. sieberiana and C. anisata as its ingredients. It is also prepared according to a recipe that has been passed down from previous generations to the current albeit with some modifications. The two plants used in this formulation are well known for their ethnomedicinal use in chronic pain management [19, 20]. In this report, we assessed the safety and effectiveness of this formulation in the larger framework of clinically validating Ghanaian herbal medicines to enhance the process of their integration into mainstream medicine and increase their uptake by conventional practitioners.

A total of 73 potential subjects were clinically evaluated for recruitment into the study. After this recruitment, 57 subjects were considered to have satisfied the selection criteria and were thus randomised into the 2 arms of the study as the intention to treat group (ITT). 


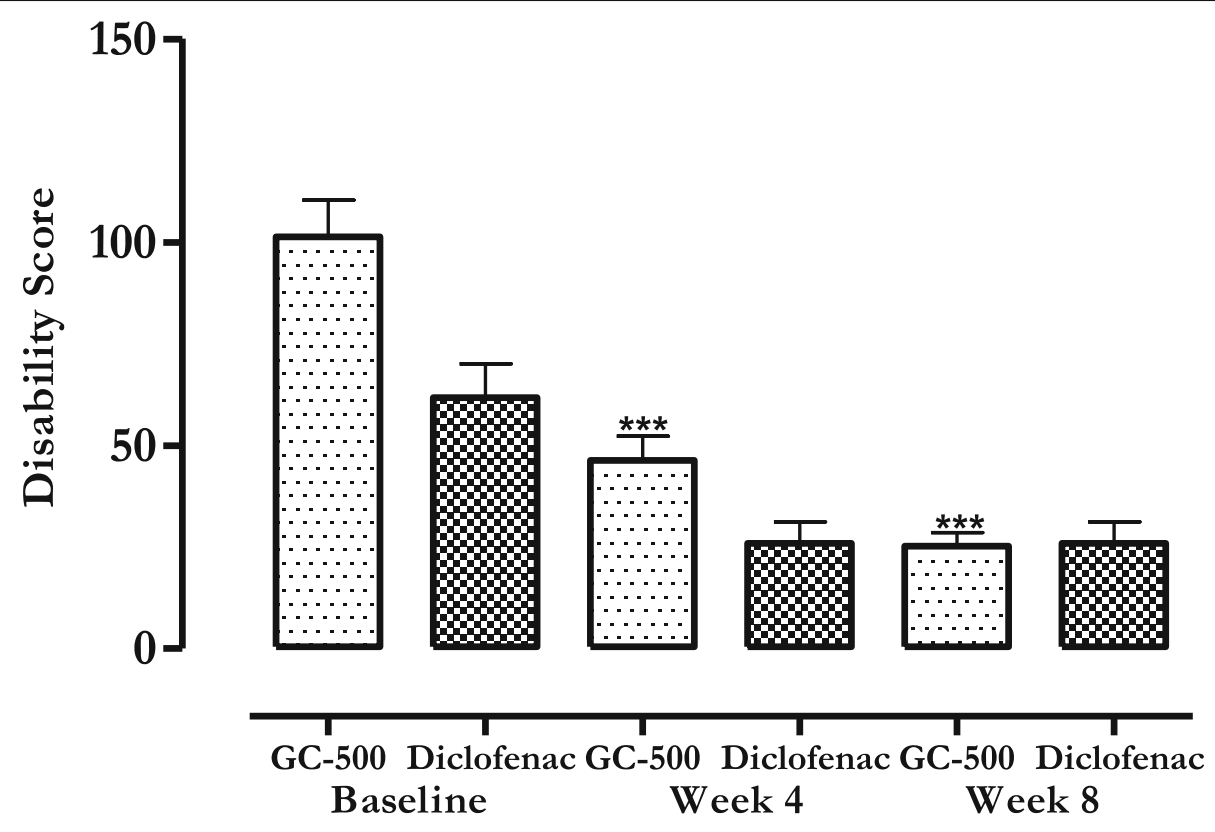

Fig. 4 The effect of GC-500 on the Disability Score of participants compared to Diclofenac. Data is expressed as Mean \pm SD. A non-significant difference was observed between the groups $p>0.05$ after ANOVA and Bonferroni post-hoc test. ${ }^{* * *} p<0.001$ : GC-500 at Week 4 \& 8 compared to its baseline

The mean age of the participants and other demographics were comparable across both arms.

Participants receiving the herbal medicine generally saw a reduction in their symptoms over the course of the 8-week study. Improvements were time dependent as the number of subjects reaching the primary outcome increased from week 4 to week 8 . An equal trend was recorded for the control group who received the standard treatment of diclofenac. At the termination of the trial, 28 (63.63\%) subjects from the GC-500 group had

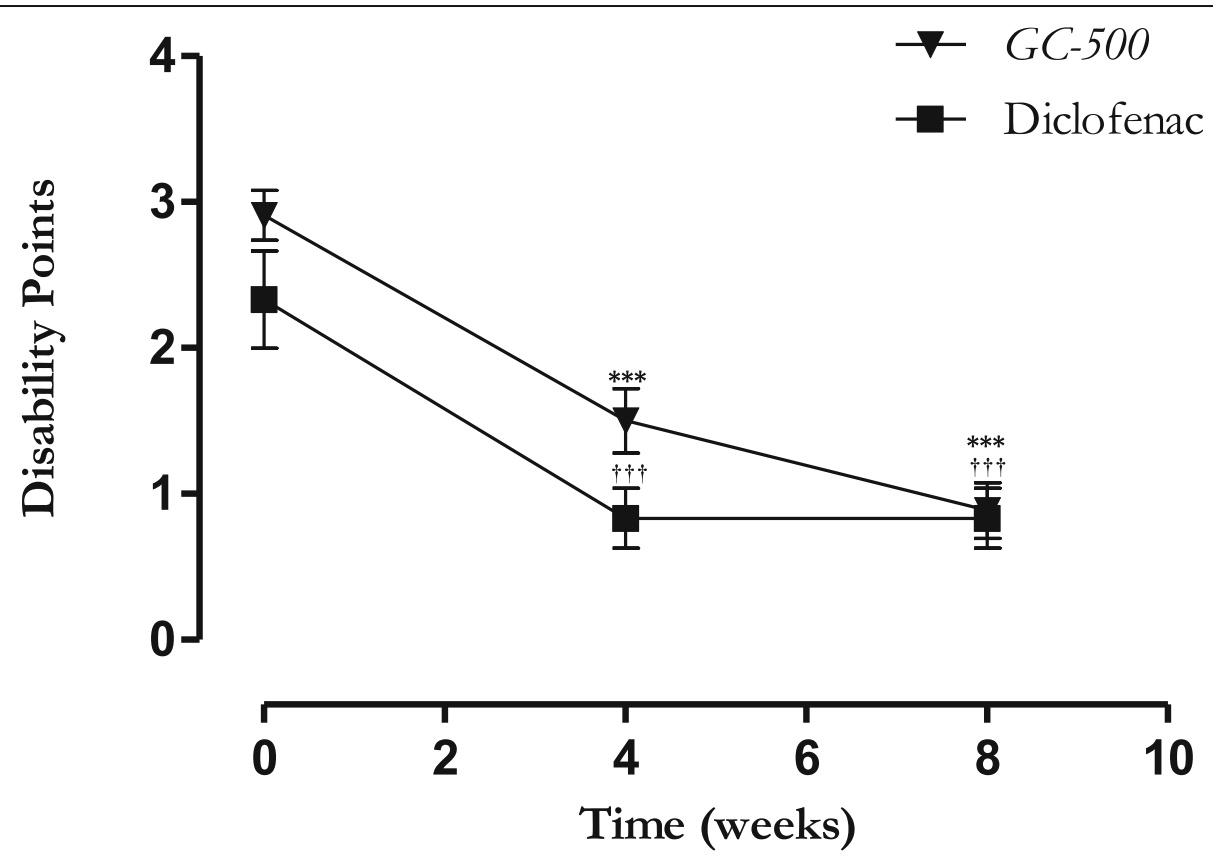

Fig. 5 Comparison of Disability Points for GC-500 and the Control over the Study Period. Data is expressed as Mean \pm SD. A non-significant difference was observed between the groups $p>0.05$ after ANOVA and Bonferroni post-hoc test. ${ }^{* * *} p<0.001$ : GC-500 at week 4 \& 8 compared to its baseline. ${ }^{\text {t十+ }} p<0.001$ : Diclofenac at week 4 \& 8 compared to its baseline 


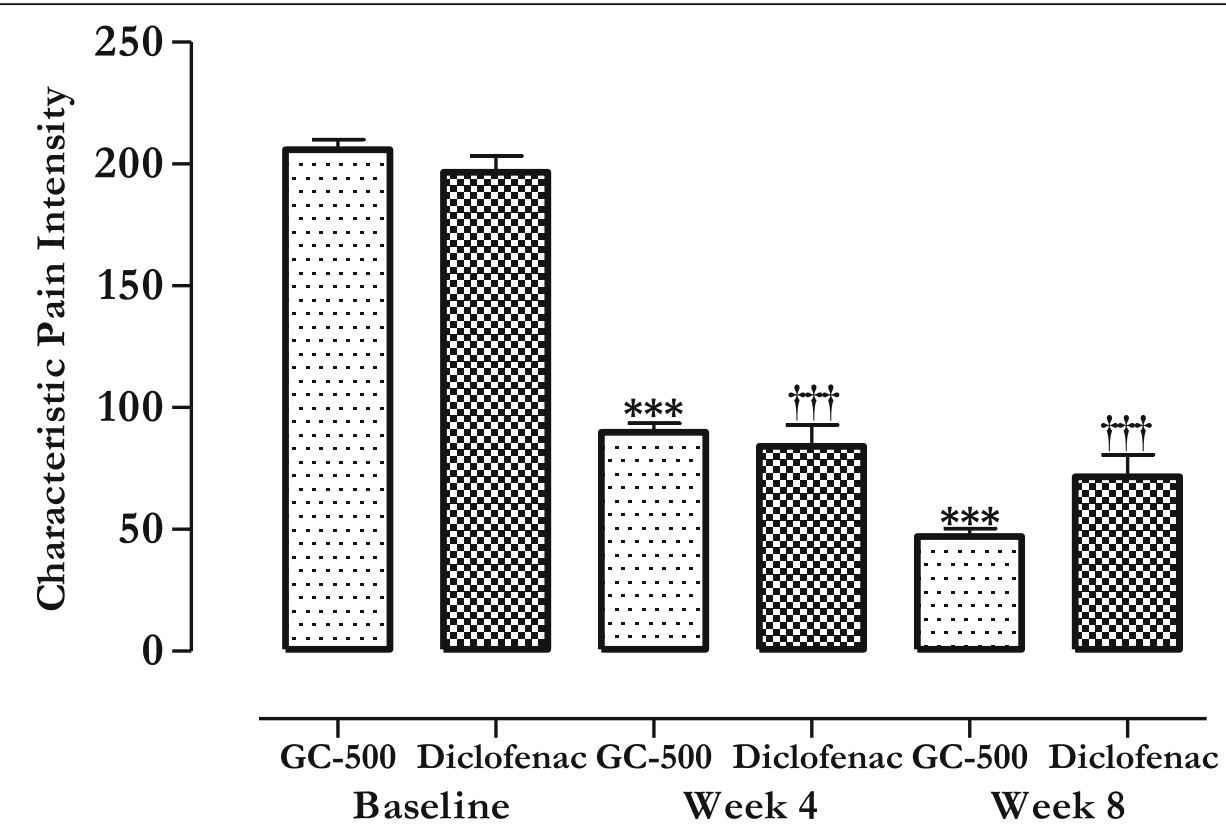

Fig. 6 The effect of GC-500 on Characteristic Pain Intensity of participants compared to Diclofenac. Data is expressed as Mean \pm SD. A nonsignificant difference was observed between the groups $p>0.05$ after ANOVA and Bonferroni post-hoc test. ${ }^{* * *} p<0.001$ : GC-500 at week 4 \& 8 compared to its baseline. ${ }^{\text {t+† }} p<0.001$ : Diclofenac at Week $4 \& 8$ compared to its baseline

reached the outcome of interest: overall treatment effect of Grade 0, compared to 5 (38.46\%) subjects from the control. Despite this variance in the achievement of the primary outcome, comparison of the effect of both treatments on the characteristic pain intensity did not indicate any statistical difference. All the participants reported of some reduction in their painful episodes and intensity confirming the analgesic and anti-inflammatory activities of the product and its components.

Clausena anisata as an analgesic and antiinflammatory agent has been widely verified. Such reports have postulated multiple mechanisms of action, but this is not unusual considering the chemical nature of medicinal herbs. Okokon and colleagues established that the leaf extract of the plant reduced ear oedema

Table 3 Biochemical Profile for GC-500 compared to the control treatment

\begin{tabular}{|c|c|c|c|c|c|}
\hline \multirow[t]{2}{*}{ Parameter } & \multicolumn{2}{|l|}{ GC-500 } & \multicolumn{2}{|l|}{ Control } & \multirow[t]{2}{*}{$\mathrm{Cl}$} \\
\hline & Baseline & End of Study & Baseline & End of Study & \\
\hline$\overline{\mathrm{AST}}(\mathrm{IU} / \mathrm{L})$ & $23.34(1.47)$ & $22.70(1.21)$ & $26.54(3.08)$ & $24.0(2.67)$ & -6.51 to 3.33 \\
\hline ALT (IU/L) & $25.98(1.25)$ & $25.23(1.47)$ & $25.51(1.65)$ & $24.82(1.93)$ & -3.90 to 5.26 \\
\hline ALP (IU/L) & $124.9(5.74)$ & $123.8(5.13)$ & $125.9(7.72)$ & $122.1(5.56)$ & -12.59 to 14.63 \\
\hline GGT (IU/L) & 18.07 (1.32) & $18.29(0.98)$ & $20.13(1.21)$ & $19.50(1.44)$ & -4.81 to 3.81 \\
\hline LDH (IU/L) & $55.87(2.02)$ & $54.71(1.97)$ & $54.84(1.33)$ & $54.60(0.98)$ & -5.78 to 6.10 \\
\hline TPROT(g/dl) & $6.89(0.17)$ & $6.93(0.13)$ & $7.20(0.14)$ & $7.04(0.12)$ & -0.61 to 0.35 \\
\hline ALB $(g / d l)$ & $3.77(0.13)$ & $3.96(0.12)$ & $3.94(0.25)$ & $3.62(0.24)$ & -0.34 to 0.60 \\
\hline TBIL ( $\mu \mathrm{mol} / \mathrm{dl})$ & $10.81(0.89)$ & $10.33(0.80)$ & $11.30(0.61)$ & $10.08(0.35)$ & -3.17 to 2.23 \\
\hline $\mathrm{Na}^{+}(\mathrm{mmol} / \mathrm{l})$ & $138.1(0.61)$ & $137.9(0.77)$ & $138.9(1.12)$ & $137.0(1.11)$ & -2.33 to 2.97 \\
\hline $\mathrm{K}^{+}(\mathrm{mmol} / \mathrm{l})$ & $4.14(0.15)$ & $4.05(0.14)$ & $4.10(0.16)$ & $3.88(0.21)$ & -0.62 to 0.44 \\
\hline $\mathrm{Cl}^{-}(\mathrm{mmol} / \mathrm{l})$ & $99.29(0.32)$ & $98.78(0.33)$ & $99.20(0.19)$ & $98.62(0.40)$ & -0.83 to 1.15 \\
\hline Urea (mmol/l) & $3.62(0.19)$ & $3.14(0.23)$ & $3.90(0.11)$ & $3.84(0.14)$ & -1.18 to .116 \\
\hline Creatinine (mmol/l) & $79.06(3.28)$ & $75.62(2.44)$ & $78.73(2.76)$ & $76.71(3.05)$ & -7.11 to 10.22 \\
\hline
\end{tabular}

Data is presented as Mean $( \pm S D) ; N=57$ : GC-500 $(n=44)$ \& Control $(n=13)$

AST Aspartate transaminase, ALT Alanine aminotransferase, ALP Alkaline phosphatase, GGT $\mathrm{Y}$-glutamyl transaminase, $\mathrm{Na}+\mathrm{Sodium}, \mathrm{K}+\mathrm{Potassium}, \mathrm{Cl}$ Chloride, $L D H$ Lactate dehydrogenase, TBIL Total bilirubin, TPROT Total protein and ALB Albumin 
Table 4 Haematological Data of Participants from both groups during the Study

\begin{tabular}{|c|c|c|c|c|c|}
\hline \multirow[t]{2}{*}{ Parameter } & \multicolumn{2}{|l|}{ GC-500 } & \multicolumn{2}{|c|}{ Dietary Control } & \multirow[t]{2}{*}{$\mathrm{Cl}$} \\
\hline & Baseline & End of Study & Baseline & End of Study & \\
\hline WBC $\left(10^{9} / \mathrm{I}\right)$ & $4.01(0.19)$ & $4.39(0.14)$ & $4.52(0.18)$ & $4.24(0.14)$ & -0.82 to 0.380 \\
\hline $\operatorname{RBC}\left(10^{12} / \mathrm{l}\right)$ & $5.15(0.23)$ & $4.77(0.17)$ & $5.15(0.23)$ & $4.77(0.17)$ & -0.87 to 0.43 \\
\hline HCT (\%) & $49.57(0.78)$ & $48.92(0.93)$ & $50.50(0.84)$ & $48.76(0.61)$ & -3.49 to 1.67 \\
\hline HGB (g/dl) & $12.91(0.91)$ & $13.44(0.12)$ & $12.93(0.16)$ & $13.11(0.18)$ & -0.16 to 0.96 \\
\hline $\operatorname{PLT}\left(10^{9} / \mathrm{l}\right)$ & $332.1(53.01)$ & $321.4(60.94)$ & $390.4(13.08)$ & $394.2(16.12)$ & - 113.6 to 19.97 \\
\hline
\end{tabular}

Data is presented as $n(\%)$ \& Mean $( \pm$ SD); $N=57$ : GC-500 $(n=44)$ \& Control $(n=13)$

WBC White Blood Cells, RBC Red Blood Cells, HCT Haematocrit, HGB Haemoglobin, PLT Platelet)

induced by xylene. This group reported the action could be linked to the inhibition of phospholipase $\mathrm{A}_{2}$ by the flavonoid group of phytochemicals. They also inferred that the ability of the plant to exert this effect may be due to its inhibition of prostaglandin $E_{2}$ through its action on the cyclooxygenase pathway and the ability to enhance the body's synthesis of endogenous antiinflammatory and anti-pyretic agents [21]. Kumatia and his co-workers also claimed the plant may work by both central and peripheral nervous pathways after their assays. They also stated the possible involvement of the opioidergic pathway as a mechanism [22].

Cassia sieberiana has also been evaluated using similar models for its efficacy and mechanism of action. The anti-inflammatory activity is said to occur through the inhibition of proinflammatory mediators such as histamine, serotonin and prostaglandins. The plant was also indicated to act by both peripheral and central nervous pathways. The involvement of opioidergic, muscarinic cholinergic and adenosinergic systems were also reported by the authors [23]. Chemically, phytochemical groups such as flavonoids, coumarins, alkaloids, sterols and triterpenes have all been said to contribute to the analgesic and anti-inflammatory properties of both plants [24-26].

Participants receiving the herbal medicinal product GC-500 also showed improvements in their disability score over time (Fig. 5). This meant a reduction in the physical and socio-economic impact of the disease. The improvement in the quality of life (QOL) also led to a significant reduction in the number of economic days lost per month, from a baseline of $0.318( \pm 0.74)$ days to $0.159( \pm 0.65)$ days $(p-0.006$; CI $0.047-0.271)$ at the end of the study. The biopsychosocial response to the treatment was summed up in the disability points for participants in both arms of the study declining significantly compared to their baseline (Fig. 5). The effect of GC-500 was again comparable to that of the standard treatment of diclofenac.

In patients with osteoarthritis, reduction in work productivity, including presenteeism, is associated with increased patient-reported severity hence the biopsychosocial response to treatment is very critical [27-29]. Poor treatment outcomes also predispose individual to altered symptoms and grave functional limitation which if not properly handled results in a cycle of pain, depression and loss of economic power [30].

Given the chronic nature of osteoarthritis, the safety of potential medicinal agents must be assured as patient exposure to these agents is lengthier. The safety profile of the herbal medicinal agent $G C-500$ was inferred to be ideal for the intended use. The product did not have any untoward effect on the liver, kidneys and haematological parameters. Baseline values were statistically not different from those obtained at the end of the study. Again, none of the participants reported of any adverse events while receiving the treatment.

The number of participants for the safety assessment was less because some subjects opted out due to aversion of needles, others for reasons that they had just recently undergone similar profiling and thus unwilling to undertake the testing again. The guidelines were relaxed to include such subjects for efficacy assessment because the formulation is not a new investigational product. The allocation ratio was increased for the herbal treatment in anticipation of this situation and also to aid in the documentation of related adverse events.

Although available reports from other groups about the toxicity of the ingredients have cited C. anisata as being safe [31], C. sieberiana has been documented as having the potential for causing detrimental effect on long-term users. Obidah and his co-workers reported that oral administration of the aqueous stem bark extract of C. sieberiana in rats resulted in hepatotoxicity at doses between 20 and $60 \mathrm{mg} / \mathrm{kg} \mathrm{BW}$ and nephrotoxicity at a dose of $180 \mathrm{mg} / \mathrm{kg} \mathrm{BW}$ [32]. This hepatotoxic effect was again observed after histopathological analysis showed the presence of necrotic and inflammatory cells in the hepatic parenchyma and a sinusoidal stasis after 28 days of treatment in rats [33]. Although, these adverse reports were from in vivo assessments, they highlight the need for the continuous process of post-market surveillance for GC-500 and most authorised herbal medicinal products to protect users. 
Notwithstanding the clinical effects of the herbal medicinal product reported here, we cannot confirm if this product has any action on the inflammatory process in osteoarthritis due to the design of the trial. The study is also limited in its ability to confirm the safety among long-term users and also the potential application of GC-500 in other osteodegenerative diseases. Our inability to blind the participants to the treatment being received could have also negatively influenced the feedback provided. This situation may be significant among the diclofenac group as the agent is first-line treatment and as such subjects may have a prior history of use.

\section{Conclusion}

The herbal medicinal product $G C-500$, provides another treatment option for managing the pain associated with osteoarthritis. The product is considered suitable because of its tolerability, improvement in subject pain scores and their quality of life all of which were comparable to diclofenac.

\section{Abbreviations}

ACN: Acetonitrile; BW: Body weight; $\mathrm{CH}_{3} \mathrm{COOH}$ : Acetic acid; GCPS: Graded Chronic Pain Scale; HPLC: High Performance Liquid Chromatography; ITT: Intention-to-treat; ICH: International Conference on Harmonisation; MEOH: Methanol; QOL: Quality of Life

\section{Acknowledgements}

The authors are indebted to the clinical staff of the out-patient unit of the Legacy Specialist Hospital, Kumasi, Ghana. We are also grateful to the Department of Herbal Medicine and the Department of Chemical Engineering, Kwame Nkrumah University of Science and Technology for assisting with the quality control of the herbal product

\section{Authors' contributions}

$K P T, J Y, A K T$ and IKA were all involved in the proposal development, trial design and the procedures leading to the ethical certification. KPT and JY undertook the patient recruitment and follow ups. KPT, AKT and IKA carried out the formulation of the herbal drug and its quality assessment. All authors were also involved in the analysis of the trial data and drafting of this manuscript.

\footnotetext{
Funding

Funding for this study was provided from the Internally Generated Funds of the Department of Herbal Medicine, Faculty of Pharmacy and Pharmaceutical Sciences, Kwame Nkrumah University of Science and Technology. Funds provided by the Department covered the manufacturing of the interventional products, running of the trial and the cost of the quality evaluation. None of the investigator received any renumeration for the research.
}

\section{Availability of data and materials}

The raw patient data used to support the findings of this study is restricted by the human experimentation committee of the Legacy Specialist Hospital, Kumasi-Ghana for protection of participant privacy. However, data is available for researchers who meet the criteria for access to confidential data from the corresponding author upon application to the Ethics Committee of the institution through the following address: Human Experimentation Committee, Legacy Specialist Hospital, PMB, Asokore-Mampong, Kumasi-Ghana.

\section{Declarations}

\section{Ethics approval and consent to participate}

The study was approved by the human experimentation committee of the Legacy Specialist Hospital (LSH/ME/002/01) and the study retrospectively registered with the Pan-African trial registry. Permission was obtained from all participants prior to recruitment.

\section{Consent for publication}

Not Applicable.

\section{Competing interests}

Authors have no conflict of interest to declare.

\section{Author details}

'Department of Pharmacognosy and Herbal Medicine, School of Pharmacy and Pharmaceutical Sciences, University of Cape Coast, Cape Coast, Ghana. ${ }^{2}$ Department of Herbal Medicine, Faculty of Pharmacy and Pharmaceutical Sciences, Kwame Nkrumah University of Science and Technology, Kumasi, Ghana. ${ }^{3}$ Department of Surgery, School of Medicine and Dentistry, Kwame Nkrumah University of Science and Technology, Kumasi, Ghana. ${ }^{4}$ Department of Biomedical Sciences, University of Cape Coast, Cape Coast, Ghana. ${ }^{5}$ Department of Pharmacognosy, Faculty of Pharmacy and Pharmaceutical Sciences, Kwame Nkrumah University of Science and Technology, Kumasi, Ghana.

Received: 31 January 2021 Accepted: 13 September 2021 Published online: 30 September 2021

\section{References}

1. Royal College of Physicians. National Collaborating Centre for Chronic Conditions. Osteoarthritis: national clinical guideline for care and management in adults. London: Royal College of Physicians; 2008. p. 3-10.

2. National Institute for Health and Care Excellence. Osteoarthritis: care and management | Guidance and guidelines. NICE CG177; 2014. p. 7-10.

3. Dziedzic KS, Healey EL, Main CJ. Implementing the NICE osteoarthritis guidelines in primary care: a role for practice nurses. Musculoskeletal Care. 2013;11(1):1-2. https://doi.org/10.1002/msc.1040.

4. Patel A, Buszewicz M, Beecham J, Griffin M, Rait G, Nazareth I, et al. Economic evaluation of arthritis self-management in primary care. BMJ (Online). 2009;339:b3532

5. Hawkins T, Barr A. Osteoarthritis: management. Clin Pharm. 2015;7:3-4

6. Kingsbury SR, Conaghan PG. Current osteoarthritis treatment, prescribing influences and barriers to implementation in primary care. Prim Health Care Res Dev. 2012;13(04):373-81. https://doi.org/10.1017/S1463423612000072.

7. Sostres C, Gargallo CJ, Arroyo MT, Lanas A. Adverse effects of non-steroidal anti-inflammatory drugs (NSAIDs, aspirin and coxibs) on upper gastrointestinal tract. Best Pract Res Clin Gastrol. 2010;24(2):121-|32. https:// doi.org/10.1016/j.bpg.2009.11.005.

8. Laine L, White WB, Rostom A, Hochberg M. COX-2 selective inhibitors in the treatment of osteoarthritis. Semin Arthritis Rheum. 2008;38(3):165-8. https:// doi.org/10.1016/j.semarthrit.2007.10.004.

9. Appiah K, Oppong C, Mardani H, Omari R, Kpabitey S, Amoatey C, et al. Medicinal plants used in the Ejisu-Juaben municipality, southern Ghana: an ethnobotanical study. Medicines. 2018;6(1):3-5. https://doi.org/10.3390/ medicines6010001.

10. Hamza OJM, van den Bout-van den Beukel CJP, Matee MIN, Moshi MJ, Mikx FHM, Selemani HO, et al. Antifungal activity of some Tanzanian plants used traditionally for the treatment of fungal infections. J Ethnopharmacol. 2006; 108(1):358-67. https://doi.org/10.1016/j.jep.2006.04.026.

11. Lawal IO, Grierson DS, Afolayan AJ. Phytochemical and antioxidant investigations of a Clausena anisata hook, a south African medicinal plant. Afr J Trad, Comp Alt Med. 2015;12(1):28-37. https://doi.org/10.4314/ajtcam. v12i1.5.

12. Sy GY, Fall AD, Diatta W, Gueye M, Badji K, Bassène E, et al. Analgesic and anti-inflammatory activity of aqueous root extract of Cassia sieberiana D. C. (Caesalpiniaceae). Afr J Pharm Pharmacol. 2009;3:651-3.

13. Aliyu Z, Yusha M, Aliyu BS. Anti malarial activity of Cassia sieberiana leaf extracts. Open Conf Proc J. 2013;4:72-6.

14. ICH. Validation of Analytical Procedures: Methodology. Rockville; 1997. https://www.fda.gov/regulatory-information/search-fda-guidance- 
documents/q2b-validation-analytical-procedures-methodology . Accessed 5 Aug 2017

15. Gul R, Jan SU, Faridullah S, Sherani S, Jahan N. Preliminary phytochemical screening, quantitative analysis of alkaloids, and antioxidant activity of crude plant extracts from Ephedra intermedia indigenous to Balochistan. Sci World J. 2017:2017:1-7. https://doi.org/10.1155/2017/5873648.

16. World Medical Association. Declaration of Helsinki: Bull. World Health Organ; 2013. p. 373-4.

17. Von Korff M, Ormel J, Keefe FJ, Dworkin SF. Grading the severity of chronic pain. Pain. 1992;50(2):133-49. https://doi.org/10.1016/0304-3959(92)90154-4.

18. Schoenfeld D. Sample Size Calculator. Boston: MGH Mallinckrodt General Clinical Research Center; 2015. http://hedwig.mgh.harvard.edu/sample_size/. Accessed 2 Nov 2017

19. Danton O, Somboro A, Fofana B, Diallo D, Sidibé L, Rubat-Coudert C, et al. Ethnopharmacological survey of plants used in the traditional treatment of pain conditions in Mali. J Herb Med. 2019;6:e05571. https://doi.org/10.1016/j. hermed.2019.100271.

20. Adebayo SA, Dzoyem JP, Shai LJ, Eloff JN. The anti-inflammatory and antioxidant activity of 25 plant species used traditionally to treat pain in southern African. BMC Comp Alt Med. 2015;15(1):159. https://doi.org/10.11 86/s12906-015-0669-5.

21. Okokon JE, Udoh AE, Andrew UE, Amazu UL. Antiinflammatory and antipyretic activities of Clausena anisata. Mol Clin Pharmcol. 2012;3:47-54.

22. Kumatia EK, Annan K, Dickson RA, Mensah AY, Amponsah IK, Appiah AA, et al. Antiinflammatory and analgesic effects in rodent models of ethanol extract of Clausena anisata roots and their chemical constituents. Nat Prod Comm. 2017;12:67-72

23. Donkor K, Okine LNK, Abotsi WKM, Woode E. Anti-inflammatory and antinociceptive effects of ethyl acetate fraction of root bark of Cassia sieberiana D. C Murine Models Pharmacologia. 2013;4(4):301-10. https://doi.org/10. 5567/pharmacologia.2013.301.310

24. Mukandiwa L, Ahmed A, Eloff JN, Naidoo V. Isolation of seselin from Clausena anisata (Rutaceae) leaves and its effects on the feeding and development of Lucilia cuprina larvae may explain its use in ethnoveterinary medicine. J Ethnopharmacol. 2013;150(3):886-91. https://doi.org/10.1016/j. jep.2013.09.037.

25. Ismail AA, Ahmad BA, Mohamed A, Rasedee A, Siddig IA, Mohamed YI, et al. A review of traditional uses, phytochemical and pharmacological aspects of selected members of Clausena genus (Rutaceae). J Med Plants Res. 2012; 6(38):5107-18. https://doi.org/10.5897/JMPR12.317.

26. Olapade AA, Ajayi OA, Ajayi IA. Physical and chemical properties of Cassia sieberiana seeds. Int Food Res J. 2014;21:767-72.

27. Sadosky A, Bushmakin A, Cappelleri J, Lionberger D. Relationship between patient-reported disease severity in osteoarthritis and self-reported pain, function, and work productivity. Arthritis Res Ther. 2010;12(4):R162. https:// doi.org/10.1186/ar3121.

28. Dieppe PA, Lohmander LS. Pathogenesis and management of pain in osteoarthritis. Lancet. 2005;365(9463):965-73. https://doi.org/10.1016/S01406736(05)71086-2

29. Hunter DJ, McDougall JJ, Keefe FJ. The symptoms of osteoarthritis and the genesis of pain. Rheum Dis Clin N Am. 2008;34(3):623-43. https://doi.org/1 0.1016/.j.rdc.2008.05.004.

30. Wilkie R, Peat G, Thomas E, Croft P. Factors associated with restricted mobility outside the home in community-dwelling adults ages fifty years and older with knee pain: an example of use of the international classification of functioning to investigate participation restriction. Arthritis Rheum. 2007:57(8):1381-9. https://doi.org/10.1002/art.23083.

31. Murungi MJ. Antimalarial activity and safety properties of Clausena anisata and Clutia robusta in a mouse model. Abst Postgrad Thesis. 2012:1:24-9.

32. Obidah W, Sa'ad UA, Wurochekke AU. Toxic effects of aqueous stem bark extract of Cassia sieberiana on some biochemical parameters in rats. Afr J Biochem Res. 2009;3:229-31.

33. Bah F, Kane Y, Fall AD, Cabral M, Toure A, Lam-Faye A, et al. Acute, subchronic toxicity in Wistar rats and cytotoxicity studies of hydroethanolic root extract of Cassia sieberiana DC. J Toxicol Pharmacol. 2017;1:17.

\section{Publisher's Note}

Springer Nature remains neutral with regard to jurisdictional claims in published maps and institutional affiliations.

\section{Submit your manuscript to a SpringerOpen ${ }^{\circ}$ journal and benefit from:}

- Convenient online submission

- Rigorous peer review

- Open access: articles freely available online

- High visibility within the field

- Retaining the copyright to your article

Submit your next manuscript at $\boldsymbol{\nabla}$ springeropen.com 